\title{
Functional renormalization group and conformal invariance in cold atoms
}

\author{
S. Moroz \\ Institut für Theoretische Physik, Philosophenweg 16, D-69120 Heidelberg, Germany
}

\begin{abstract}
We present an application of a field-theoretic functional renormalization group technique to fewbody (vacuum) physics of nonrelativistic atoms near a Feshbach resonance. We consider and compare a onecomponent bosonic system with a two-component fermionic system, focusing on the scale-free unitarity limit of an infinite scattering length. While the two-body problem is consistent with nonrelativistic conformal symmetry for the two considered systems, a conformal anomaly, manifested through a renormalization group limit cycle with the Efimov parameter $s_{0} \approx 1.006$, is found for bosons in the three-body problem.
\end{abstract}

\section{Introduction}

Physics of ultracold atoms is an exciting area of research which advances rapidly both experimentally and theoretically (for reviews see [1]). This is mainly due to the excellent experimental control and tunability of the studied systems. In particular, the two-body interaction strength of atoms near a so-called Feshbach resonance can be changed in broad ranges by applying an external magnetic field. This fact makes these systems an ideal simulator of various theoretical models at strong coupling.

Near a broad Feshbach resonance the low-energy fewbody physics becomes universal: some physical observables are insensitive to the detailed form of the microscopic interaction potential and depend only on the s-wave scattering length $a$. The unitarity limit is of a particular theoretical interest because all length scales drop out of the problem, and the theory becomes classically scale (and even nonrelativistic conformal) invariant. In addition, in this limit the theory is strongly coupled and nonperturbative treatment is necessary.

It is a theoretically well-established result, derived first by Efimov in [2], that a discrete energy spectrum of shallow three-body bound states develops in the quantum threebody problem of resonantly interacting bosons. At unitarity, the spectrum is infinite and geometric, meaning that the ratio of energies of the adjacent levels is a constant, independent of the level number. For a long time this counterintuitive effect was a pure theoretical prediction with no experimental realization. However, the remarkable recent experiment with ultracold cesium atoms by Kraemer et al. [3] provided the first evidence that the Efimov effect can be realized in Nature. The finding of Kraemer et al. stimulated extensive experimental activity [4-6].

In this work we present our studies of the two- and three-body problem for fermions and bosons near a broad Feshbach resonance. We use a field-theoretic functional renormalization group method and focus our attention to

\footnotetext{
a e-mail: s.moroz@thphys.uni-heidelberg.de
}

the issue of conformal invariance and its quantum anomaly in the nonrelativistic physics of cold atoms.

\section{Functional renormalization group}

We use quantum field theory methods to investigate the nonrelativistic few-body physics of cold atoms. In particular, we employ a functional renormalization group (FRG) method which proved to be fruitful for studies of various many-body problems (for reviews see [7]). Here we shortly summarize our approach.

In quantum field theory a central object of interest is the Schwinger functional $W[J]$. In Euclidean formulation the exponential of this functional can be schematically written as a functional integral

$$
e^{W[J]}=\int \mathscr{D} \varphi e^{-S[\varphi]+\int J \varphi},
$$

where $\varphi$ collectively represents different fields coupled to the external sources $J$. The microscopic action $S[\varphi]$ is assumed to be known and defines the theory in the ultraviolet (UV). The Schwinger functional is a generator of connected correlation functions, i.e. one can construct $n$ point connected Green functions by functional differentiation of $W[J]$ with respect to the sources $J$. Using the LSZ reduction formula and the spectral decomposition of correlation functions, it is possible to extract physical quantities such as scattering amplitudes and bound state energies from Green functions. The straightforward prescription, described above, is hindered by the fact that the functional integral in Eq. (1) is difficult to evaluate in a generic interacting quantum field theory. To tackle this problem we use a renormalization group (RG) idea and introduce into Eq. (1) a regulator term (cut-off) $R_{k}$ which depends on a sliding scale $k$. The Schwinger functional acquires scale dependence and is now determined by

$$
e^{W_{k}[J]}=\int \mathscr{D} \varphi e^{-S[\varphi]-\frac{1}{2} \int \varphi R_{k} \varphi+\int J \varphi} .
$$




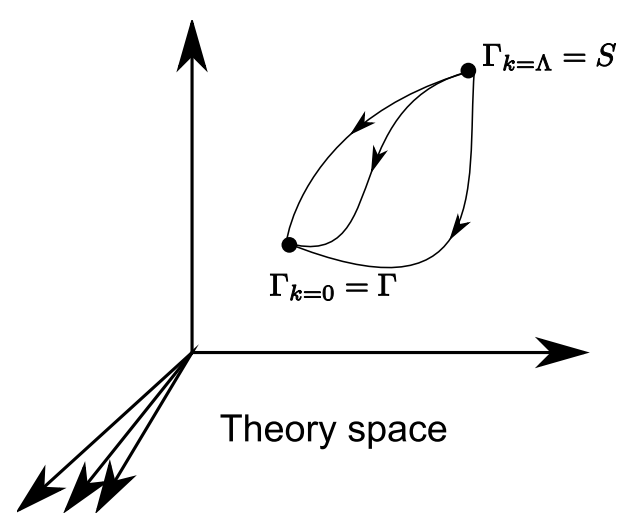

Fig. 1. Renormalization group flow in the theory space of all possible couplings allowed by symmetries. Different trajectories correspond to different choices of the infrared regulator $R_{k}$.

In the spirit of the Wilsonian renormalization group, we would like to investigate how the Schwinger functional $W_{k}[J]$ depends on the sliding scale $k$. More precisely, we will study $k$-dependence of a flowing action functional $\Gamma_{k}[\phi]$ which is the central object in the FRG method. The flowing action $\Gamma_{k}[\phi]$ is defined as a modified Legendre transformation of $W_{k}[J]$, i.e.

$$
\Gamma_{k}[\phi]=\sup _{J}\left(\int J \phi-W_{k}[J]\right)-\frac{1}{2} \int \phi R_{k} \phi .
$$

The reason why one is interested in $\Gamma_{k}[\phi]$ instead of $W_{k}[J]$ is that the former solves a compact functional differential equation

$$
\partial_{k} \Gamma_{k}[\phi]=\frac{1}{2} \mathrm{~S} \operatorname{Tr} \frac{\partial_{k} R_{k}}{\Gamma_{k}^{(2)}[\phi]+R_{k}},
$$

derived first by Wetterich in [8]. Here STr stands for a generalized supertrace which sums over momenta, Matsubara frequencies, internal indices and fields (taking fermions with a minus sign) and the second functional derivative $\Gamma_{k}^{(2)}[\phi]$ denotes the full inverse regulated field propagator. The renormalization group evolution of $\Gamma_{k}[\phi]$, prescribed by the Wetterich equation (4), can be illustrated in the theory space, which is a space of all possible couplings allowed by symmetries of the problem. As schematically shown in Fig. 1, at the microscopic UV scale $k=\Lambda$ one starts with $\Gamma_{k=\Lambda}=S$. As the sliding scale $k$ is lowered, the flowing action $\Gamma_{k}$ evolves in the theory space according to Eq. (4). The choice of the regulator $R_{k}$ is by no means unique, which introduces some scheme dependence into the problem. For this reason, different choices of the regulator $R_{k}$ correspond to the different paths in Fig. 1. At the infrared (IR) scale $k=0$, however, one should recover the full effective action $\Gamma_{k=0}=\Gamma$ for every choice of the cutoff $R_{k}$, and all trajectories should meet at the same point in Fig. 1 .

In practice, it is a difficult task to solve Eq. (4) exactly. One is forced to introduce a physically motivated expansion of the flowing action $\Gamma_{k}$ and perform a truncation of the Wetterich equation. Nevertheless, the expansions do not necessarily involve a small parameter, and they are in general of nonperturbative nature.

\section{The models: bosons vs fermions}

As advocated in $[9,10]$, a systematic vertex expansion of $\Gamma_{k}$ is especially suitable for studies of few-body problems. The vertex expansion is an expansion in powers of fields, i.e.

$$
\Gamma_{k}=\sum_{n=0}^{\infty} \Gamma_{k}(n)=\Gamma_{k}(2)+\Gamma_{k}(3)+\Gamma_{k}(4)+\ldots
$$

where $n$ represents the number of fields in the monomial $\Gamma_{k}(n)$. In the second equation $\Gamma_{k}(0)$ and $\Gamma_{k}(1)$ are missing because with no loss of generality the vacuum state energy can be shifted to zero, and the term linear in fields is absent by construction.

We are interested in the few-body physics of neutral atoms interacting via a Feshbach resonance, which can be described by a two-channel model. In particular, we introduce and compare two different systems:

- One-component bosons- Our expansion of the flowing action $\Gamma_{k}$ expressed in the Fourier space reads

$$
\begin{aligned}
\Gamma_{k}(2)= & \int_{Q} \psi^{*}(Q) P_{\psi}(Q) \psi(Q)+\int_{Q} \varphi^{*}(Q) P_{\varphi}(Q) \varphi(Q), \\
\Gamma_{k}(3)= & \frac{1}{2} \int_{Q_{1}, Q_{2}} h\left(Q_{1}, Q_{2}\right)\left[\varphi^{*}\left(Q_{1}\right) \psi\left(Q_{2}\right) \psi\left(Q_{1}-Q_{2}\right)\right. \\
& \left.+\varphi\left(Q_{1}\right) \psi^{*}\left(Q_{2}\right) \psi^{*}\left(Q_{1}-Q_{2}\right)\right], \\
\Gamma_{k}(4)= & -\int_{Q_{1}, \ldots Q_{3}} \lambda_{3}\left(Q_{1}, Q_{2}, Q_{3}\right) \\
& \times \varphi\left(Q_{1}\right) \psi\left(Q_{2}\right) \varphi^{*}\left(Q_{3}\right) \psi^{*}\left(Q_{1}+Q_{2}-Q_{3}\right),
\end{aligned}
$$

where $Q=(\omega, \mathbf{q})$ and $\int_{Q}=\int_{-\infty}^{\infty} \frac{d \omega}{2 \pi} \int_{-\infty}^{\infty} \frac{d^{3} \mathbf{q}}{(2 \pi)^{3}}$. The field $\psi$ denotes an elementary complex bosonic atom, while $\varphi$ represents a complex bosonic composite diatom which mediates the Feshbach interaction. The vacuum system enjoys Galilean space-time symmetry and an internal $U(1)$ symmetry which reflects conservation of the particle number of elementary atoms. The Yukawa coupling $h$ can be related to the width of the Feshbach resonance. In addition, we introduce into our truncation the atom-diatom vertex $\lambda_{3}\left(Q_{1}, Q_{2}, Q_{3}\right)$, assumed to vanish in the UV.

- Two-component fermions- The truncation of the flowing action $\Gamma_{k}$ for this system is given by

$$
\begin{aligned}
\Gamma_{k}(2)= & \sum_{i=1}^{2} \int_{Q} \psi_{i}^{*} P_{\psi}(Q) \psi_{i}(Q)+\int_{Q} \varphi^{*}(Q) P_{\varphi}(Q) \varphi(Q), \\
\Gamma_{k}(3)= & -\int_{Q_{1}, Q_{2}} h\left(Q_{1}, Q_{2}\right)\left[\varphi^{*}\left(Q_{1}\right) \psi_{1}\left(Q_{2}\right) \psi_{2}\left(Q_{1}-Q_{2}\right)\right. \\
& \left.-\varphi\left(Q_{1}\right) \psi_{1}^{*}\left(Q_{2}\right) \psi_{2}^{*}\left(Q_{1}-Q_{2}\right)\right],
\end{aligned}
$$


$19^{\text {th }}$ International IUPAP Conference on Few-Body Problems in Physics
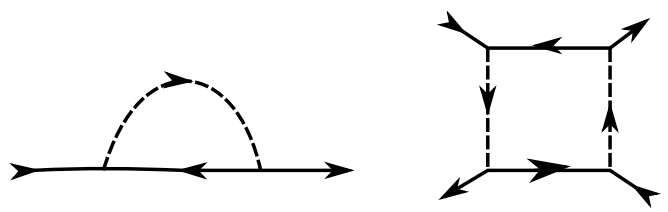

Fig. 2. Two examples of the Feynman diagrams which vanish in the nonrelativistic vacuum. Dashed lines denote diatoms $\varphi$, while solid lines represent atoms $\psi$.

$$
\begin{aligned}
\Gamma_{k}(4)= & \int_{Q_{1}, \ldots Q_{3}} \lambda_{3}\left(Q_{1}, Q_{2}, Q_{3}\right) \\
& \times \sum_{i=1}^{2} \varphi\left(Q_{1}\right) \psi_{i}\left(Q_{2}\right) \varphi^{*}\left(Q_{3}\right) \psi_{i}^{*}\left(Q_{1}+Q_{2}-Q_{3}\right),
\end{aligned}
$$

where the Grassmann-valued fields $\psi_{1}$ and $\psi_{2}$ represent two species of elementary fermionic atoms, and $\varphi$ denotes a bosonic composite diatom. At the UV scale $\varphi \sim \psi_{1} \psi_{2}$. This model has Galilean space-time symmetry and an internal $S U(2) \times U(1)$ symmetry.

\section{Vacuum state and nonrelativistic conformal invariance}

Although the FRG method allows us to study different manybody problems at finite temperature (for example, see [11]), here we are solely interested in the few-body (vacuum) physics. Specifically, our aim is to study excitations above the nonrelativistic vacuum state, i.e. above the state with zero density $n=0$ and zero temperature $T=0$. As was shown in $[9,10]$, numerous mathematical simplifications appear in the nonrelativistic vacuum compared with the many-body state. For instance, all Feynman diagrams with loop lines pointing in the same direction vanish. Two simple examples of this type of diagrams are depicted in Fig. 2. Physically, this simplification originates from a fact that there are only particles, but no holes in the nonrelativistic vacuum. Mathematically, this can be demonstrated by employing the residue theorem for the frequency integration. In addition, a remarkable hierarchy of RG flow equations holds in the nonrelativistic vacuum. The hierarchy emerges from the observation that the flow equations of the n-body sector $^{1}$ are not influenced by the couplings from the higherbody sectors. We note that exactly the presence of this hierarchy makes the systematic vertex expansion so useful for the treatment of few-body problems. The generally applicable strategy thus is to solve n-body problems subsequently by starting with the lower sectors and proceeding to the higher ones.

The quantum-mechanical few-body systems, discussed here, posses a centrally extended Galilean space-time symmetry. The centrally extended Galilean algebra in three

\footnotetext{
1 The $n$-body sector is defined as a set of $2 n$-points $1 \mathrm{PI}$ vertices, expressed in terms of elementary fields.
}

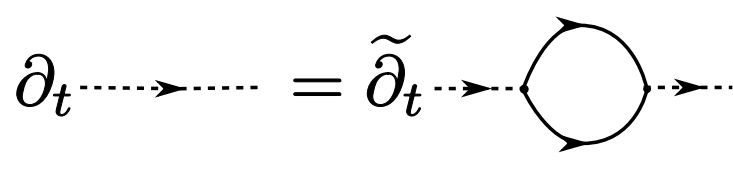

Fig. 3. RG flow of the diatom inverse propagator $P_{\varphi}(Q)$ in terms of Feynman diagrams. Dashed lines denote diatoms $\varphi$, while solid lines represent atoms $\psi$. On the LHS the scale derivative $\partial_{t} \equiv k \frac{d}{d k}$, whereas on the right-hand-side $\widetilde{\partial}_{t}$ acts only on the regulator $R_{k}$ terms in the loop.

spatial dimensions consists of 11 generators: particle number $N$ (central charge), time translation $H$, three spatial translations $P_{i}$, three spatial rotations $M_{i j}$ and three Galilean boosts $K_{i}$. It is known for a long time, however, that space-time symmetries of a free Schrödinger equation form a larger symmetry group, the so-called Schrödinger (or nonrelativistic conformal) group [12]. The Schrödinger algebra extends the Galilei algebra by two additional generators: the nonrelativistic dilation generator $D$ and the special conformal generator $C$. It is remarkable that there are some examples of interacting theories which exhibit nonrelativistic conformal invariance at quantum level [13, 14]. These theories are called nonrelativistic conformal field theories (NRCFT), and Schrödinger symmetry put additional powerful constraints on the form of correlation functions in these systems. In the following, by studying the two- and three-body problem, we address the question whether the bosonic and fermionic models, introduced above, are examples of NRCFTs in the classically scale invariant (unitarity) regime.

\section{One- and two-body problems}

Due to the presence of the vacuum hierarchy, we first solve the one-body sector by calculating the RG evolution of the inverse atom propagator $P_{\psi}(Q)$. As it turns out, it is not renormalized in the bosonic and fermionic systems and keeps its microscopic value

$$
P_{\psi}(Q)=i \omega_{\mathbf{q}}+\mathbf{q}^{2}+E_{\psi}
$$

Here our units are $\hbar=k_{B}=2 M_{\psi}=1$, where $M_{\psi}$ is the mass of the atom. In addition, $E_{\psi}$ denotes a gap parameter of the elementary field $\psi$.

We turn our attention to the two-body sector, i.e. to the Yukawa coupling $h\left(Q_{1}, Q_{2}\right)$ and the inverse diatom propagator $P_{\varphi}(Q)$ in the two considered models. We find that the Yukawa coupling does not flow, i.e. $\partial_{k} h\left(Q_{1}, Q_{2}\right)=0$. The RG flow of the inverse propagator $P_{\varphi}(Q)$ is schematically shown in Fig. 3. The flow equation for $P_{\varphi}(Q)$ can be solved analytically $[9,10]$, and the solution for bosons and fermions differs only by a simple multiplicative factor. Keeping in mind the question asked in the end of Sec. 4 we present here the solution only in the simple, but physically interesting unitarity regime, where the inverse s-wave scattering length $a^{-1} \rightarrow 0$ and the Feshbach resonance is broad $h^{2} \rightarrow \infty$. In this regime all scales drop out, and the physics 
becomes completely universal. At unitarity the atom and diatom inverse propagators read

$$
P_{\psi}(Q)=i \omega_{\mathbf{q}}+\mathbf{q}^{2}, \quad P_{\varphi}(Q) \sim \sqrt{\frac{i \omega_{\mathbf{q}}}{2}+\frac{\mathbf{q}^{2}}{4}} .
$$

We observe that the diatom field $\varphi$ acquires a nontrivial scaling dimension $[\varphi]=2$, compared with the trivial (Gaussian) scaling $[\psi]=\frac{3}{2}$ of the atom field. The inverse propagators $P_{\psi}$ and $P_{\varphi}$ of the fermionic and bosonic system are gapless and thus respect full Schrödinger symmetry in the unitarity regime.

\section{Three-body problem at unitarity}

Here we tackle a more complex three-body problem and present a solution of the FRG equation for the atom-diatom 1PI vertex $\lambda_{3}\left(Q_{1}, Q_{2}, Q_{3}\right)$. The flow of $\lambda_{3}\left(Q_{1}, Q_{2}, Q_{3}\right)$ is schematically illustrated in Fig. 4. Fortunately, it is possible to reduce the number of arguments of the atom-diatom vertex by transforming to the center-of-mass frame, putting the energies of the incoming and outgoing atoms on-shell and restricting to a domain of low energies and momenta. The kinematic reduction reads

$$
\lambda_{3}\left(Q_{1}, Q_{2}, Q_{3}\right) \rightarrow \lambda_{3}\left(q_{1}, q_{2}, E\right),
$$

where $q_{1}\left(q_{2}\right)$ is the magnitude of the incoming (outgoing) particles and $E$ is the total collision energy. After this simplification the RG flow equation for the atom-diatom vertex can be derived. In Minkowski space-time it is given by $^{2}$

$$
\begin{aligned}
& \partial_{t} \lambda_{3}\left(q_{1}, q_{2}, E\right)=-\frac{8}{\pi} \frac{k^{3}}{\sqrt{\frac{3 k^{2}}{4}-\frac{E}{2}-i \epsilon}} \\
& \times\left[C \lambda_{3}\left(q_{1}, k, E\right) \lambda_{3}\left(k, q_{2}, E\right)+A G\left(q_{1}, k\right) G\left(k, q_{2}\right)\right. \\
& \left.+B / 2\left\{\lambda_{3}\left(q_{1}, k, E\right) G\left(k, q_{2}\right)+G\left(q_{1}, k\right) \lambda_{3}\left(k, q_{2}, E\right)\right\}\right]
\end{aligned}
$$

with

$$
G\left(q_{1}, q_{2}\right)=\frac{1}{4 q_{1} q_{2}} \log \frac{q_{1}^{2}+q_{2}^{2}+q_{1} q_{2}-\frac{E}{2}-i \epsilon}{q_{1}^{2}+q_{2}^{2}-q_{1} q_{2}-\frac{E}{2}-i \epsilon} .
$$

The coefficients $A, B$ and $C$ for bosons and fermions can be found in Table 1 .

In order to understand basic features of the RG evolution of the three-body coupling $\lambda_{3}\left(q_{1}, q_{2}, E\right)$, we first make a crude pointlike approximation $\lambda_{3}\left(q_{1}, q_{2}, E\right) \rightarrow \lambda_{3}(E) \rightarrow$ $\lambda_{3}$, i.e. we neglect the momentum dependence of the atomdiatom coupling and take the low-energy limit $E \rightarrow 0$. It is clear from Eq. (11) that the generic form of the RG equation now reads

$$
\partial_{t} \lambda_{3 R}=\alpha \lambda_{3 R}^{2}+\beta \lambda_{3 R}+\gamma, \quad \alpha, \beta, \gamma \in \mathbb{R},
$$

${ }^{2}$ In the three-body sector calculation we first integrate out atom fluctuations by lowering the cut-off $R_{\psi}$, while keeping $R_{\varphi}$ fixed. Subsequently in the second step, we use a sharp cut-off for the diatoms and lower $R_{\varphi}$ to zero.
Table 1. Numerical coefficients $A, B$, and $C$ in the flow equation (11) for the two examined systems.

\begin{tabular}{lccc}
\hline Model & A & B & C \\
\hline Bosons & 1 & 2 & 1 \\
Fermions & 1 & -2 & 1 \\
\hline
\end{tabular}

where we introduced the rescaled dimensionless coupling $\lambda_{3 R}=k^{2} \lambda_{3}$. The form of the solution of Eq. (13) is determined by the sign of the discriminant $D=\beta^{2}-4 \alpha \gamma$ of the quadratic polynomial on the right-hand-side of Eq. (13). For $D>0$ the $\beta$-function has two real roots (Fig. 5) and the RG flow towards the IR interpolates between the UV and IR fixed points (Fig. 5). On the other hand, for $D<0$ the $\beta$-function has no real roots (Fig. 5), and the RG flow of $\lambda_{3 R}$ is governed by an infinite (unbounded) limit cycle (Fig. 5).

For the fermionic system (7) one obtains $D>0$. Hence, for fermions at unitarity the three-body coupling $\lambda_{3 R}$ flows towards a fixed point and thus respects full Schrödinger symmetry. For bosons one gets $D<0$, and the solution is completely different. The intuitive interpretation of the limit cycle solution is that during the RG flow we hit threebody atom-diatom bound states, which manifest themselves as divergences of $\lambda_{3 R}$. In the unitary limit there are infinitely many of these bound states, which are equidistant on the logarithmic scale. The continuous scaling symmetry is broken to the discrete $Z$ group. This is the well-known Efimov effect [2]. In the case of equivalent bosons the Efimov result is

$$
\frac{E_{n+1}}{E_{n}}=\exp \left(-2 \pi / s_{0}\right)
$$

with $E_{n+1}$ and $E_{n}$ denoting energies of neighboring bound states. The Efimov parameter $s_{0}$ is given by the solution of a transcendental equation and one finds $s_{0} \approx 1.006$ [2]. By dimensional arguments we can connect the artificial sliding scale $k^{2}$ with the scattering energy $E$ as $E \sim k^{2}$. The proportionality factor disappears in the ratio of the energies and hence the Efimov parameter can be read off from the RG period $T$

$$
\frac{k_{n+1}^{2}}{k_{n}^{2}}=\frac{E_{n+1}}{E_{n}}=\exp (-2 T) \Rightarrow s_{0}=\frac{\pi}{T} .
$$

It is remarkable that for the two considered systems the complicated flow equation (11) at $E=0$ can be solved exactly [10], and the result coincides with the solution of the Skorniakov-Ter-Martirosian integral equation [15]. For illustration, we present a numerical solution of Eq. (11). A series of cartoons of the evolution of the reduced 1PI vertex $^{3} f_{t}\left(t_{1}, t_{2}\right) \equiv 4(3+p) q_{1} q_{2} \lambda_{3}\left(q_{1}, q_{2}, E=0\right)$ for both systems is shown in Fig. 6. For fermions, first a peak appears in the UV $\left(t_{1}=0, t_{2}=0\right)$, which propagates in the diagonal direction $\left(t_{1}=t_{2}\right)$ during the RG evolution. On the other hand, for bosons, a periodic structure (with

\footnotetext{
${ }^{3}$ Here we prefer to work with logarithms of momenta $t_{1}=$ $\ln \left(q_{1} / \Lambda\right)$ and $t_{2}=\ln \left(q_{2} / \Lambda\right)$. Additionally, we use $p=+1$ for bosons and $p=-1$ for fermions.
} 


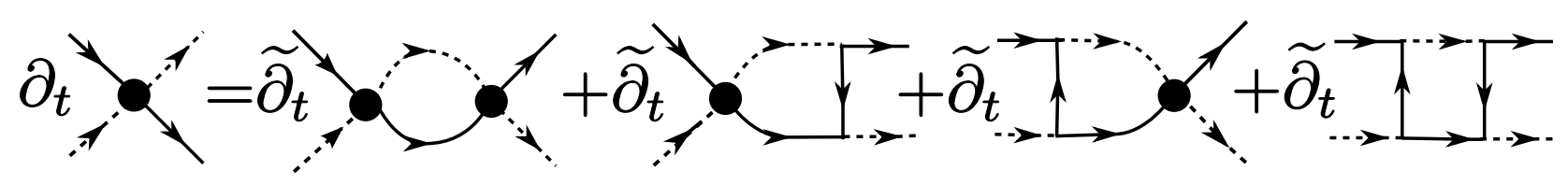

Fig. 4. Feynman diagram representation of the RG flow of the atom-diatom coupling $\lambda_{3}\left(Q_{1}, Q_{2}, Q_{3}\right)$ illustrated by the dark blob. Dashed lines denote diatoms, while solid lines represent atoms. On the LHS the scale derivative $\partial_{t} \equiv k \frac{d}{d k}$, whereas on the right-hand-side $\widetilde{\partial}_{t}$ acts only on the regulator $R_{k}$ terms in the loops.
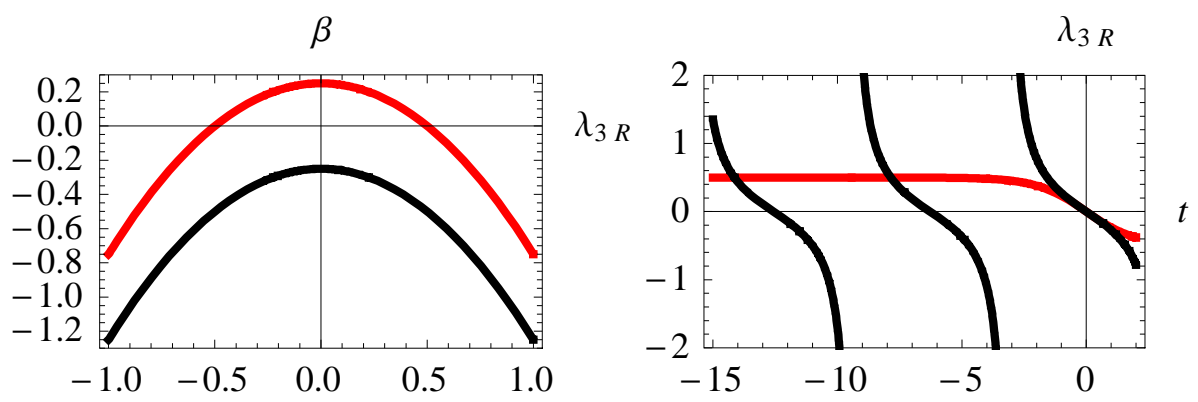

Fig. 5. The $\beta$-function (left) and the RG flow of the coupling $\lambda_{3 R}$ as a function of the logarithmic sliding scale $t$ (right) for bosons (black line) and fermions (red line).

period $T_{\text {spatial }} \approx 6.2$ in both directions) develops gradually. The evolution in the RG "time" $t$ of the UV point $f_{t}\left(t_{1}=0, t_{2}=0\right)$ for both systems is depicted in Fig. 7 . While for fermions the evolution is monotonic in time, in case of bosons we obtain a "temporal" oscillation of pe$\operatorname{riod} T_{\text {temp }} \approx 3.1$. For different points in the $t_{1}-t_{2}$ plane the "time" evolution is triggered at the scale $t_{\text {in }} \sim O\left(t_{1}, t_{2}\right)$.

The numerical solution for bosons is consistent with the results of $[16,17]$. Spatial and temporal oscillations are correlated. The Efimov parameter $s_{0}$ can be calculated as $s_{0}=\frac{\pi}{T_{\text {temp }}} \approx 1.0$, which is in a good agreement with the Efimov result $s_{0} \approx 1.00624$.

Finally, in addition to the two systems discussed above, another interesting model of three-component fermions with $S U(3) \times U(1)$ internal symmetry was studied in [10]. It was demonstrated that this system exhibits the Efimov effect with the Efimov parameter $s_{0}$ identical to the bosonic case. Furthermore, the three-component fermions were studied with FRG in [18] using an elegant three-channel model, where a three-particle composite trion field is explicitly introduced. This approach was utilized in [19] to provide theoretical description of results of recent experiments with three-component ${ }^{6} \mathrm{Li}$ fermionic atoms $[4,5]$.

\section{Conclusion and outlook}

In conclusion, we demonstrated how the FRG method can be used to study few-body physics of ultracold bosons and fermions near a Feshbach resonance. In the renormalization group language the two-body problem at unitarity is governed by a nongaussian fixed point, and both (bosonic and fermionic) systems seem to be examples of nonrelativistic conformal field theories. In the three-body problem the situation is different for the two considered systems: in the theory of two-component fermions the atom-diatom coupling $\lambda_{3 R}$ flows toward the IR fixed point, while no IR fixed point exists in the bosonic theory. For bosons, the atom-diatom RG flow undergoes a quantum scale anomaly and exhibits the RG limit cycle behavior, which is a manifestation of the Efimov effect.

In future it will be very interesting to study four- and higher-body sectors of the $S U(2)$ fermionic theory at unitarity with FRG and test the conjecture, made in [13,14], that this particular theory is a real example of a nonrelativistic conformal field theory in all n-body sectors. In addition, the generalization of the Efimov effect for four (and more) bosons at unitarity is of a great theoretical and experimental interest. First steps towards the FRG treatment of the four-boson problem have been undertaken recently in [20].

\section{Acknowledgments}

The presented work was done in calloboration with S. Floerchinger, R. Schmidt and C. Wetterich. The author is supported by KTF. 

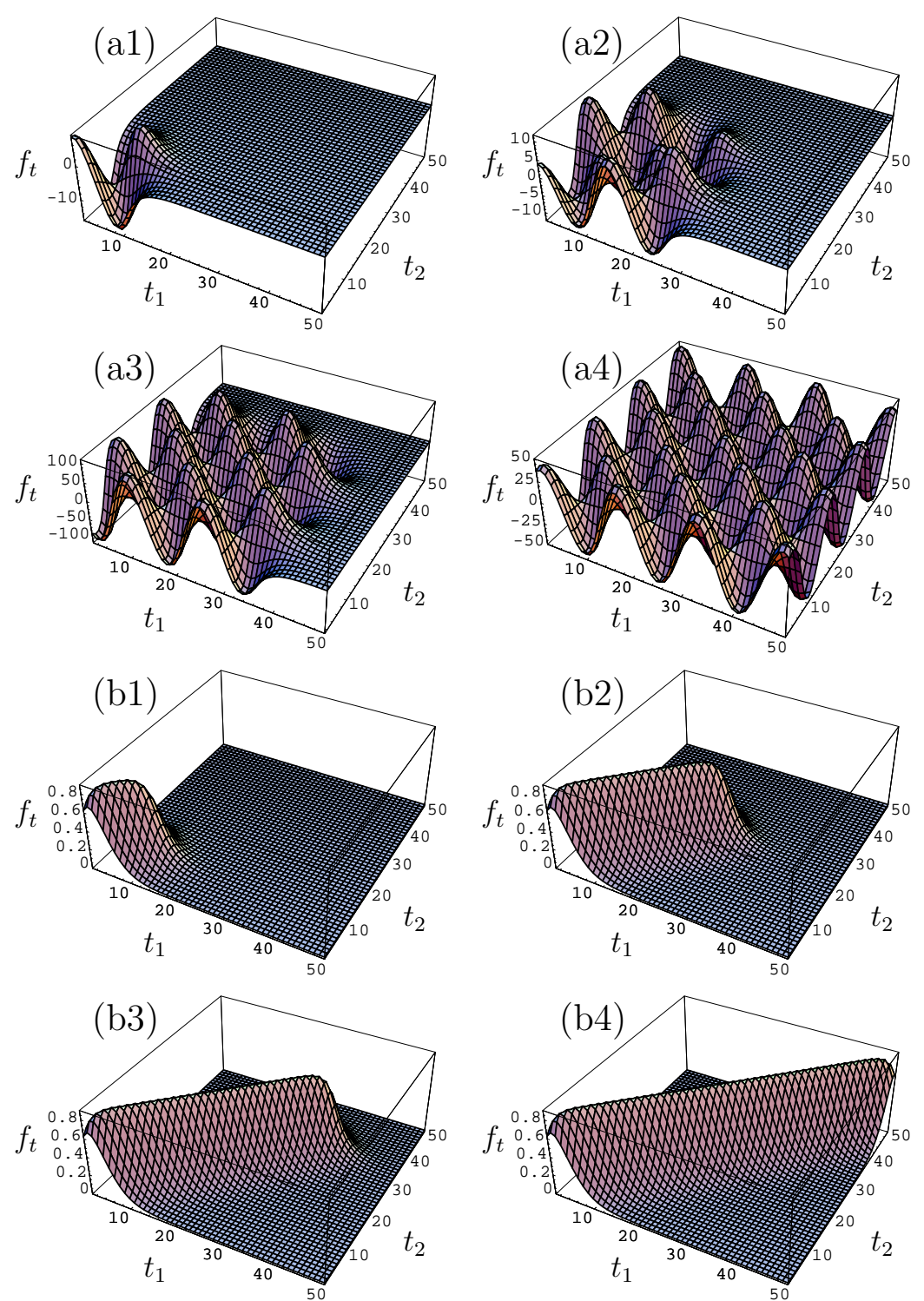

Fig. 6. The RG evolution of the momentum dependent modified vertex $f_{t}\left(t_{1}, t_{2}\right)=4(3+p) q_{1} q_{2} \lambda_{3}\left(q_{1}, q_{2}, E=0\right)$ for bosons (a1-a4) and SU(2) fermions (b1-b4). Spatial momenta $t_{1}, t_{2}$ and the RG "time" $t$ are descritized to $N=50$ intervals with a step $\Delta t=0.4$. The cartoons for bosons and fermions correspond to the descritized steps $10,25,35,50$.
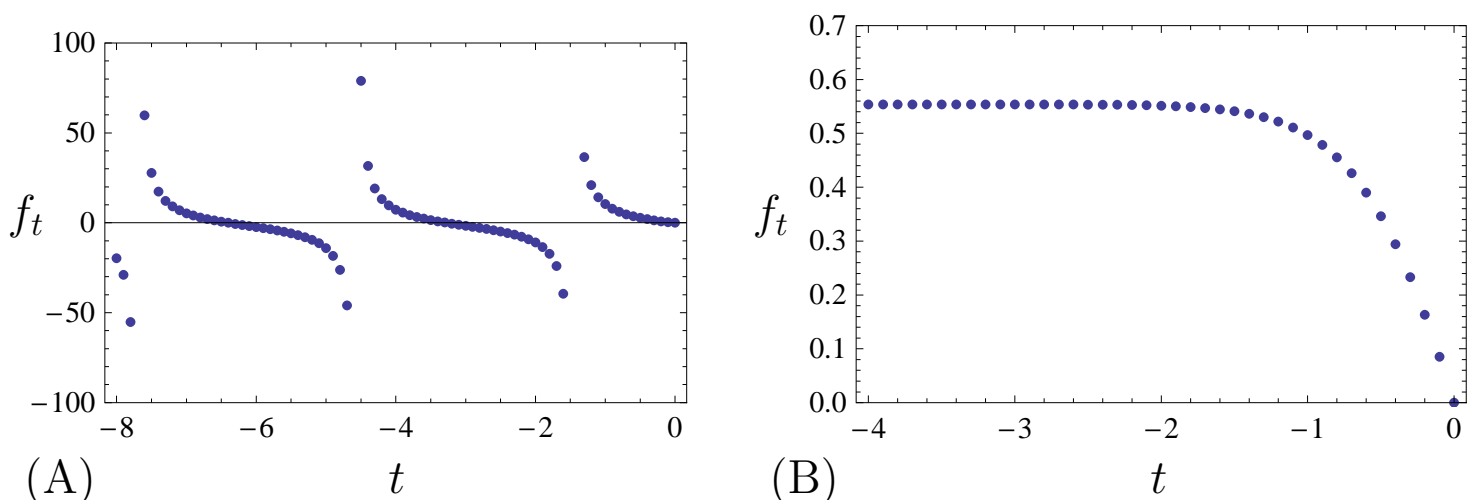

Fig. 7. Numerical evolution in the RG "time" $t$ of $f_{t}\left(t_{1}=0, t_{2}=0\right)$ for bosons (A) and fermions (B). For SU(2) fermions (B) the modified vertex approaches a fixed point solution; in the case of bosons (A), a limit cycle behavior is developed with a period $T_{\text {temp }} \approx 3.1$. 
$19^{\text {th }}$ International IUPAP Conference on Few-Body Problems in Physics

\section{References}

1. I. Bloch, J. Dalibard and W. Zwerger, Rev. Mod. Phys. 80, 885 (2008); S. Giorgini, L. P. Pitaevskii and S. Stringari, Rev. Mod. Phys. 80, 1215 (2008).

2. V. Efimov, Phys. Lett. 33B, 563 (1970); V. Efimov, Nucl. Phys. A 210, 157 (1973).

3. T. Kraemer et al., Nature 440, 315 (2006).

4. T. B. Ottenstein et al., Phys. Rev. Lett. 101, 203202 (2008).

5. J. H. Huckans et al., Phys. Rev. Lett. 102, 165302 (2009); J. R. Williams et al., Phys. Rev. Lett. 103, 130404 (2009).

6. S. Knoop et al., Nature Phys. 5, 277 (2009); M. Zaccanti et al., Nature Phys. 5, 586 (2009); G. Barontini et al. Phys. Rev. Lett. 103, 043201(2009); F. Ferlaino, et al., Phys. Rev. Lett. 102, 140401 (2009); S. Pollack, D. Dries, R. Hullet, arXiv:0911.0893 [cond-mat].

7. J. Berges, N. Tetradis and C. Wetterich, Phys. Rept. 363 (2002) 223; T. R. Morris, Prog. Theor. Phys. Suppl. 131 (1998) 395; K. Aoki, Int. J. Mod. Phys. B 14 (2000) 1249; C. Bagnuls and C. Bervillier, Phys. Rept. 348 (2001) 91; J. Polonyi, Central Eur. J. Phys. 1 (2003) 1; M. Salmhofer and C. Honerkamp, Prog. Theor. Phys. 105 (2001) 1; B. Delamotte, D. Mouhanna and M. Tissier, Phys. Rev. B 69 (2004) 134413; B. J. Schaefer and J. Wambach, hep$\mathrm{ph} / 0611191$.

8. C. Wetterich, Phys. Lett. B 301 (1993) 90.

9. S. Diehl, H. C. Krahl, M. Scherer, Phys. Rev. C 78, 034001 (2008).

10. S. Moroz, S. Floerchinger, R. Schmidt and C. Wetterich, Phys. Rev. A 79, 042705 (2009).

11. S. Diehl, S. Floerchinger, H. Gies, J. M. Pawlowski and C. Wetterich, arXiv:0907.2193 [cond-mat.quantgas].

12. C. R. Hagen, Phys. Rev. D 5, 377 (1972); U. Niederer, Helv. Phys. Acta 45, 802 (1972).

13. T. Mehen, I. W. Stewart and M. B. Wise, Phys. Lett. B 474, 145 (2000).

14. Y. Nishida and D. T. Son, Phys. Rev. D 76, 086004 (2007).

15. G. V. Skorniakov, K. A. Ter-Martirosian, Zh. Eksp. Teor. Phys. 31, 775 (1956), [Sov. Phys. JETP 4, 648 (1957)].

16. P. F. Bedaque, H. W. Hammer and U. van Kolck, Phys. Rev. Lett. 82, 463 (1999).

17. P. F. Bedaque, H. W. Hammer and U. van Kolck, Nucl. Phys. A 646, 444 (1999).

18. S. Floerchinger, R. Schmidt, S. Moroz, and C. Wetterich, Phys. Rev. A 79, 013603 (2009).

19. S. Floerchinger, R. Schmidt, and C. Wetterich, Phys. Rev. A 79, 053633 (2009).

20. R. Schmidt and S. Moroz, arXiv:0910.4586 [condmat]. 